Supplementary Materials for

\title{
Deterministic Light Yield, Fast Scintillation, and Microcolumn Structures in Lead Halide Perovskite Nanocrystals
}

Francesco Maddalena ${ }^{1,2, *}$, Aozhen Xie ${ }^{1,2}$, Xin Yu Chin ${ }^{3}$, Raihana Begum ${ }^{3}$, Marcin E. Witkowski ${ }^{4}$, Michal Makowski ${ }^{4}$, Benoit Mahler ${ }^{5}$, Winicjusz Drozdowski ${ }^{4}$, Stuart Victor Springham ${ }^{6}$, Rajdeep Singh Rawat ${ }^{6}$, Nripan Mathews ${ }^{3}$, Christophe Dujardin ${ }^{5}$, Muhammad Danang Birowosuto ${ }^{1,2, *}$, and Cuong Dang ${ }^{1,2, *}$

${ }^{1}$ School of Electrical and Electronic Engineering, Nanyang Technological University, 50 Nanyang Avenue, 639798, Singapore

${ }^{2}$ CINTRA UMI CNRS/NTU/THALES 3288, Research Techno Plaza, 50 Nanyang Drive, Border X Block, Level 6, 637553, Singapore

${ }^{3}$ Energy Research Institute @ NTU, 50 Nanyang Drive, X-Frontiers Block, Level 5, 637553, Singapore

${ }^{4}$ Institute of Physics, Faculty of Physics, Astronomy, and Informatics, Nicolaus Copernicus University in Torun, ul. Grudziadzka 5, 87-100 Torun, Poland

${ }^{5}$ Universitéé de Lyon, Université Claude Bernard, Lyon 1, CNRS, Institut Lumière Matière UMR5306, Villeurbanne F-69622, France

${ }^{6}$ Natural Sciences and Science Education, National Institute of Education, 1 Nanyang Walk, 637616, Singapore

*Corresponding Authors. Email: francesco_maddalena@ntu.edu.sg; mbirowosuto@ntu.edu.sg; hcdang@ntu.edu.sg;

Keywords: perovskite, nanocrystals, X-ray, $\gamma$-ray. 


\section{List of supplementary materials}

Figure S1. X-ray attenuation lengths for all investigated lead halide perovskite (LHP) nanocrystals (NCs).

Figure S2. A sampling of the LHP NCs-covered glass substrates that were used during the characterization.

Figure S3. Distribution of the NCs-sizes of the investigated materials.

Table S1. The photoluminescence (PL), X-ray excited luminescence (XL), band gap and Stoles' Shift of the investigated perovskite halide NCs.

Figure S4. Pulse-height spectra of the investigated perovskite halide NCs.

Table S2. Afterglow decay time and residual XL at $10 \mathrm{~K}$ of the examined perovskite halide NCs.

Figure S5. Thermoluminescence (TL) peaks for the investigated perovskite halide NCs and the corresponding fits.

Table S3. Parameters of the TL peak fitting.

Table S4. XL decay time of the investigated perovskite halide NCs. 


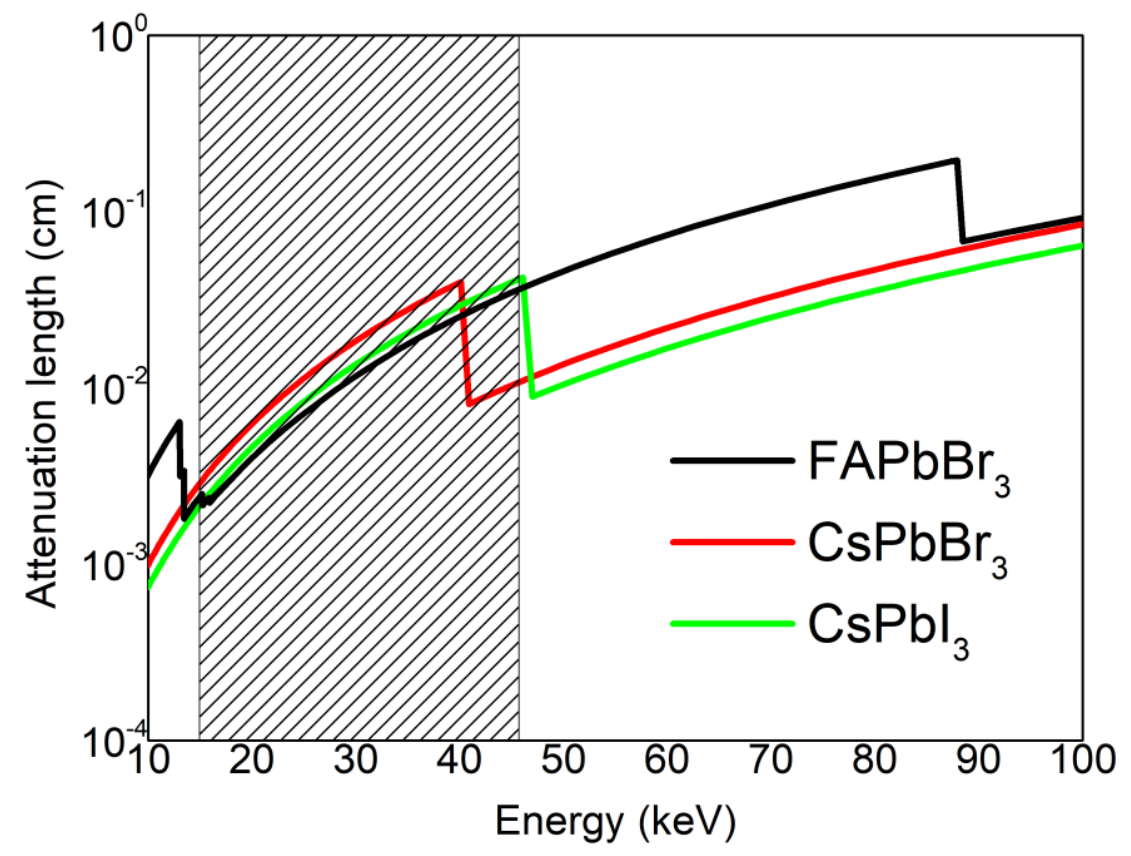

Figure S1. X-ray attenuation lengths for all investigated NCs. The area with diagonal patterns is indicated for the X-ray energies between 15 and $45 \mathrm{keV}$.

\section{$\mathrm{CsPbBr}_{3}$}

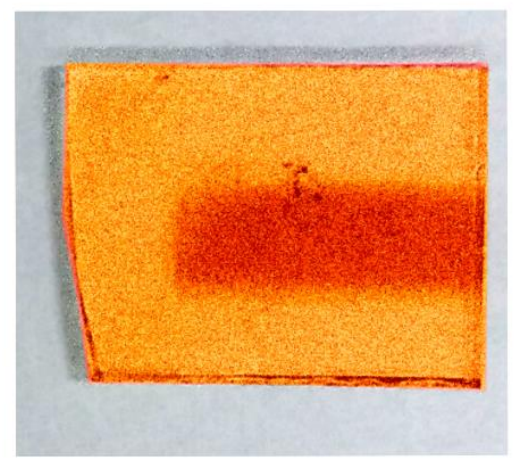

\section{$\mathrm{FAPbBr}_{3}$}

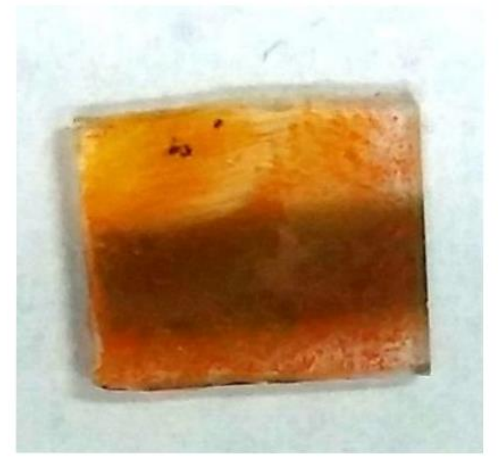

$\mathrm{CsPbl}_{3}$

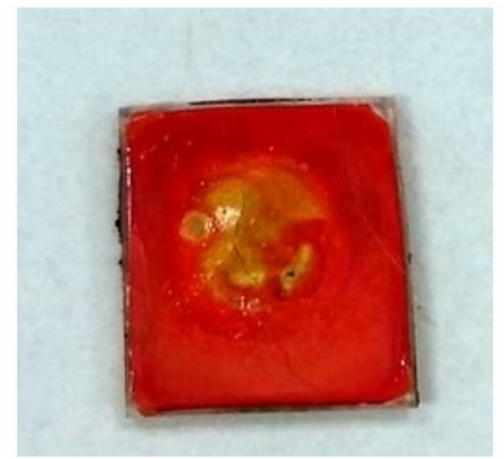

Figure S2. A sampling of the LHP NCs-covered glass substrates that were used for characterization. The darker/damaged areas represent the area of prolonged exposure to X-ray excitation. 

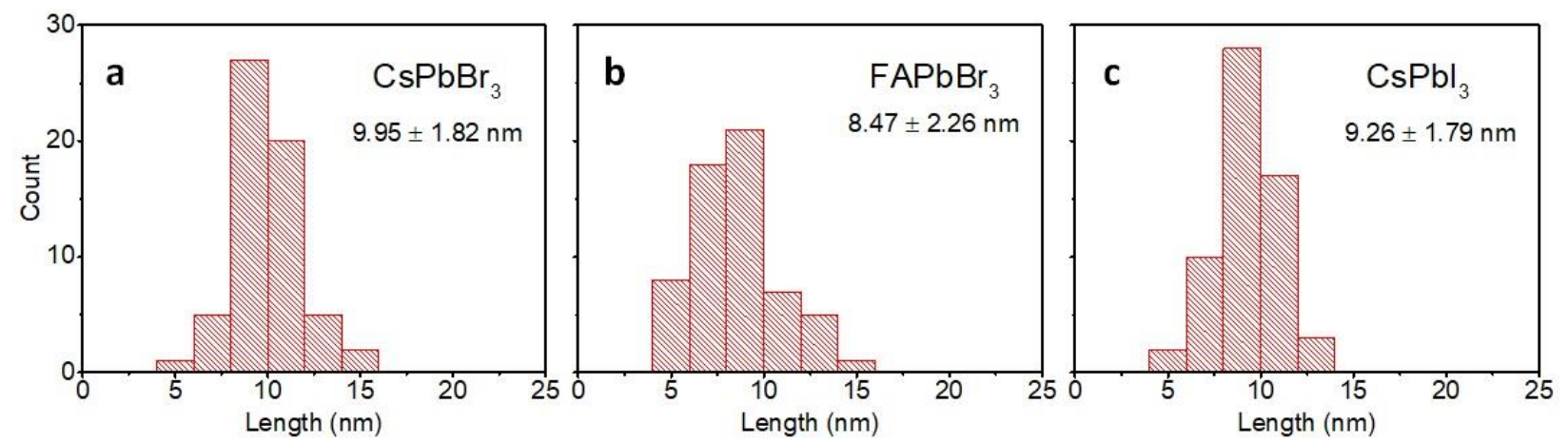

Figure S3. Distribution of $\mathrm{NC}$ sizes for a) $\mathrm{CsPbBr}_{3}$, b) $\mathrm{FAPbBr}_{3}$, and c) $\mathrm{CsPbI}_{3}$.

Table S1. The photoluminescence (PL), X-ray excited luminescence (XL), band gap and Stokes Shift of the investigated perovskite halide NCs.

\begin{tabular}{|l|l|l|l|l|}
\hline Compound & $\begin{array}{l}\text { PL Peak } \\
(\mathbf{n m})\end{array}$ & $\begin{array}{l}\text { XL Peak } \\
(\mathbf{n m})\end{array}$ & $\begin{array}{l}\text { Band gap } \\
(\mathbf{e V})\end{array}$ & $\begin{array}{l}\text { Stokes Shift } \\
(\mathbf{n m})\end{array}$ \\
\hline $\mathrm{CsPbBr}_{3}$ & 517 & 524 & 2.40 & 37 \\
\hline $\mathrm{FAPbBr}_{3}$ & 545 & 550 & 2.11 & 28 \\
\hline $\mathrm{CsPb}_{3}$ & 667 & 667 & 1.76 & 3 \\
\hline
\end{tabular}




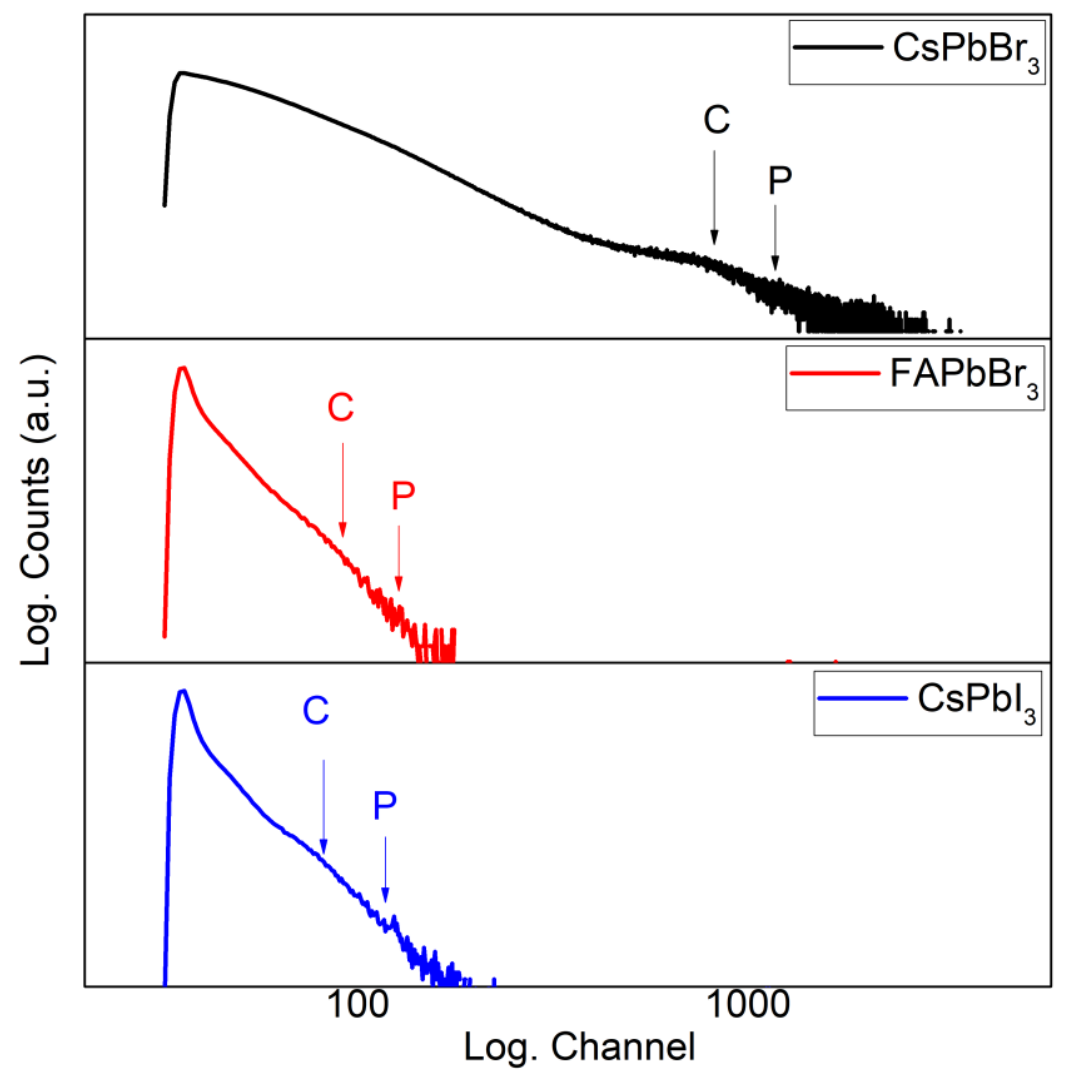

Figure S4. Pulse-height spectra of the investigated perovskite halide NCs, using a ${ }^{137}$ Cs source $(662 \mathrm{keV})$. C indicates the Compton edge and $\mathrm{P}$ the photopeak positions.

Table S2. Afterglow decay time and residual XL after $5 \mathrm{~s}$ at $10 \mathrm{~K}$ of the examined perovskite halide NCs, where $\tau_{i}$ is the exponential afterglow decay time component, $C_{i}$ is the percentage weight of the component and $\bar{\tau}$ is the average afterglow decay time.

\begin{tabular}{|l|l|l|l|}
\hline Compound & $\boldsymbol{\tau}_{\boldsymbol{i}}(\mathbf{s})\left[\boldsymbol{C}_{\boldsymbol{i}}\right]$ & $\overline{\boldsymbol{\tau}}(\mathbf{s})$ & Residual XL after 5s \\
\hline $\mathrm{CsPbBr}_{3}$ & $25.3[43.0 \%] ; 128[32.8 \%] ; 619[24.2 \%]$ & 202 & $5.2 \%$ \\
\hline $\mathrm{FAPbBr}_{3}$ & $15.0[30.2 \%] ; 78.9[49.5 \%] ; 366[20.3 \%]$ & 118 & $0.38 \%$ \\
\hline $\mathrm{CsPb}_{3}$ & $19.5[64.0 \%] ; 160[36.0 \%]$ & 70.3 & $0.80 \%$ \\
\hline
\end{tabular}




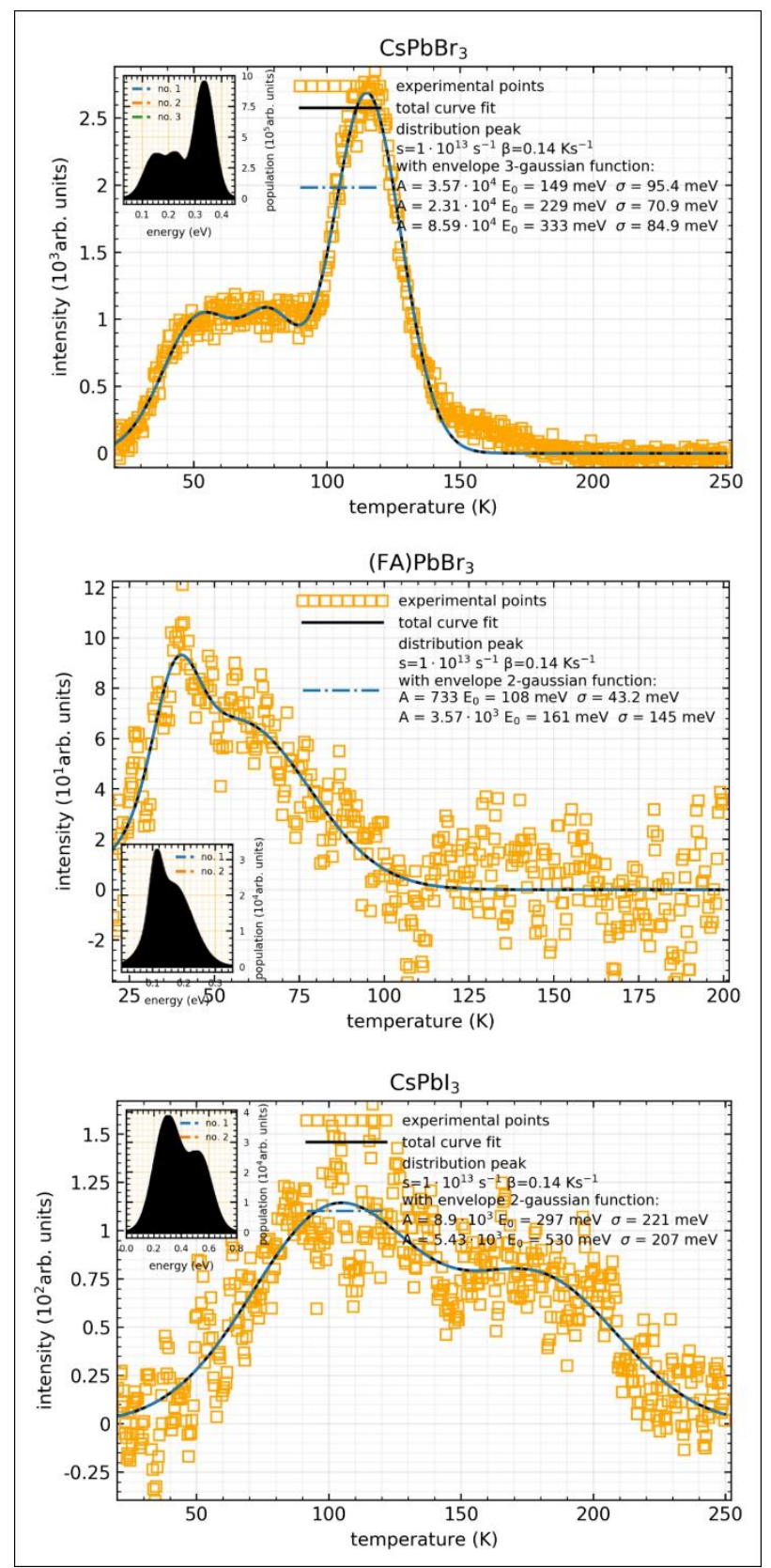

Figure S5. Thermoluminescence (TL) peaks for the investigated perovskite halide NCs and the corresponding fits.

Glow peak fits. We assume that all of traps which are occurring in TL spectra can be represented as quasi-continuous trap distributions. In order to determine the trap parameters (including quasicontinuous trap distributions), we use the procedure described by Brylew et al. ${ }^{1}$ assuming arbitrary value of the frequency factor for distribution at $10^{13} \mathrm{~s}^{-1}$. The example of the fit procedures is shown in Fig. S3 while the traps parameters obtained from the fitting procedure are given in Table S3. 
Table S3. Parameters of the TL peak fitting, where $T_{\max }$ is temperature where the maximum of the peak occurs, $E$ is the trap depth, and $n_{0}$ is the trap concentration. A frequency factor of $10^{13} \mathrm{~s}^{-1}$ was used for all fittings. ${ }^{1}$

\begin{tabular}{|l|l|l|l|}
\hline Sample & $\begin{array}{l}T_{\max } \\
(\mathrm{K})\end{array}$ & $\begin{array}{l}E \\
(\mathrm{meV})\end{array}$ & $\begin{array}{l}n_{0} \\
(\text { a.u. })\end{array}$ \\
\hline \multirow{2}{*}{$\begin{array}{l}\mathrm{CsPbBr} \\
\mathrm{NCs}\end{array}$} & 54 & 149 & $3.57 \times 10^{4}$ \\
\cline { 2 - 4 } & 78 & 229 & $2.31 \times 10^{4}$ \\
\cline { 2 - 4 } & 118 & 333 & $8.59 \times 10^{4}$ \\
\hline \multirow{2}{*}{$\begin{array}{l}\mathrm{FAPbBr}_{3} \\
\mathrm{NCs}\end{array}$} & 38 & 108 & $7.33 \times 10^{2}$ \\
\cline { 2 - 4 } & 56 & 161 & $3.57 \times 10^{3}$ \\
\hline \multirow{2}{*}{$\begin{array}{l}\mathrm{CPPb}{ }_{3} \\
\mathrm{NCs}^{2}\end{array}$} & 100 & 297 & $8.90 \times 10^{3}$ \\
\cline { 2 - 4 } & 171 & 530 & $5.43 \times 10^{3}$ \\
\hline
\end{tabular}

Table S4. XL decay time of the investigated perovskite halide NCs, where $\tau_{i}$ is the exponential decay time component, $C_{i}$ is the percentage weight of the component and $\bar{\tau}$ is the average decay time.

\begin{tabular}{|l|l|l|}
\hline Compound & $\boldsymbol{\tau}_{\boldsymbol{i}}(\mathbf{n s})\left[\boldsymbol{C}_{\boldsymbol{i}}\right]$ & $\overline{\boldsymbol{\tau}}(\mathbf{n s})$ \\
\hline $\mathrm{CsPbBr}_{3}$ & $1.41[26.7 \%] ; 5.15[49.3 \%] ; 12.7[24.0 \%]$ & 5.97 \\
\hline $\mathrm{FAPbBr}_{3}$ & $1.70[24.2 \%] ; 6.52[32.8 \%] ; 37.7[43.0 \%]$ & 18.7 \\
\hline $\mathrm{CsPbI}_{3}$ & $0.51[75.6 \%] ; 2.45[23.2 \%] ; 12.5[1.2 \%]$ & 1.10 \\
\hline
\end{tabular}

\section{References}

1. Brylew, K.; Drozdowski, W.; Wojtowicz, A. J.; Kamada, K.; Yoshikawa, A., Studies of low temperature thermoluminescence of GAGG:Ce and LuAG:Pr scintillator crystals using the Tmax-Tstop method. J. Lumin. 2014, 154, 452-457. 